\title{
COMPORTAMIENTO CÍCLICO EN LAS IMPORTACIONES Y EXPORTACIONES CUBANAS: UN ANÁLISIS DESDE EL DOMINIO DE LAS FRECUENCIAS
}

\author{
CYCLIC BEHAVIOR IN CUBAN IMPORTS AND EXPORTS: \\ AN ANALYSIS FROM THE FREQUENCY DOMAIN
}

\author{
${ }^{1}$ Orlando Ramírez Stout, ${ }^{2}$ Nancy Quińones Chang \\ ${ }^{1}$ Facultad de Economia, Universidad de La Habana, La Habana Cuba \\ ${ }^{2}$ Instituto Técnico Militar José Martí, La Habana Cuba.
}

\section{RESUMEN}

El propósito de este trabajo es estudiar las periodicidades o ciclos detectables en las series de las importaciones y exportaciones cubanas, haciendo uso del análisis espectral. En las últimas dos décadas, la economía cubana ha experimentado cambios como la inserción del sector privado y la inversión extranjera. Por lo tanto, es de interés conocer el comportamiento recurrente de la actividad económica en el corto plazo. La idea de identificar ciclos detectables en lugar de realizar un estudio completo de los ciclos económicos de la actividad se fundamenta en que las series disponibles no son muy extensas y un análisis de periodicidades de largo período requiere muestras estadísticas grandes. El objetivo planteado aquí es mucho más modesto. Se requiere identificar movimientos particularmente en períodos cortos y obtener información precisa sobre tales periodicidades.

Palabras clave: análisis espectral, series de tiempo económicas.

\section{Abstract}

This paper presents the application of spectral analysis in order to find cycles components of two economical series from Cuba today. In the last two decades, the Cuban economy has experienced changes like the insert of the private sector and the foreign investment. Therefore it is of interest to know the recurrent behavior of the economic activity in the short period. The idea of identifying cycles instead of carrying out a complete study of the economic cycles of the activity is based in that the available series are not very extensive and a of periodicities' analysis of long period requires large statistical samples. The objective here is much more modest, only to identify movements particularly in short periods and to obtain precise information on such cycles.

Keywords: economical time series, spectral analysis.

\footnotetext{
${ }^{1}$ orlando@fec.uh.cu

2 nancyqefec.uh.cu
} 


\section{INTRODUCCIÓN}

Considerando la utilidad del ciclo para evaluar y diseñar políticas económicas y teniendo en cuenta que el ciclo es un

componente inobservable, por lo que no es posible realizar pruebas de ajuste para sus estimaciones; es muy provechoso contar con varios métodos para la extracción de la señal cíclica en una serie temporal. Otro aspecto esencial de este análisis es la identificación de ciclos predominantemente determinísticos con período y fase constante (Jaramillo, 2010). En economía generalmente predominan los ciclos de carácter estocásticos. Sin embargo, la estimación de comportamientos determinísticos puede ser considerada una manera de estilizar e interpretar comportamientos más o menos estables (Granger, 1964). Estos elementos configuran una hipótesis de trabajo para modelar comportamientos recurrentes duraderos.

Las series de importaciones y exportaciones constituyen indicadores macroeconómicos que forman parte del PIB; pero que por sí mismos contienen valor informativo de interés sobre el devenir económico del país; por lo cual identificar la existencia de ciclos en las mismas constituye un paso inicial para posteriores estudios.

\section{Materiales y MÉTODOS}

Las series cronológicas empleadas en el presente trabajo corresponden al valor monetario de los volúmenes anuales de las importaciones y exportaciones del Estado cubano entre los años 1950 y 2014; están expresados en millones de pesos y los mismos son publicados por la Oficina Nacional de Estadísticas e Información (ONEI).

\section{Análisis espectral}

A continuación, serán expuestos de forma breve los principales resultados teóricos para el cálculo del espectro, para detalles y demostraciones matemáticas ver (Cohen, 1999; Hamilton, 1994; Priestley, 1981).
En esencia, el método espectral descompone una serie de tiempo estacionaria como una suma de un conjunto de series de componentes cíclicas con propiedades específicas. Se debe considerar que este tipo de análisis nunca va a estar en contradicción con los resultados generados por los modelos ARIMA (González, 2001); aunque es importante resaltar el hecho de que el análisis espectral no depende de un modelo para generar resultados. Este analiza la serie en forma puramente matemática y no está basado en ninguna teoría acerca de los procesos que definen las series. Por esto se requiere de la mayor cantidad de datos posibles para utilizar esta técnica.

$\mathrm{Al}$ análisis espectral se le llama también análisis en el dominio de frecuencia, ya que la frecuencia es una medida para representar ciclos. La frecuencia es el número de ciclos por unidad de tiempo. A continuación, se describen los aspectos básicos del análisis espectral para una serie de tiempo y se puntualizan las principales ventajas y desventajas del uso de esta técnica.

La idea análisis espectral es que un proceso estacionario $\boldsymbol{Y}_{t}$ puede ser descrito como la suma de movimientos de seno y coseno de diferentes frecuencias y amplitudes. La meta es determinar cuáles son los ciclos de diferentes frecuencias importantes para describir el comportamiento de $\boldsymbol{Y}_{\boldsymbol{t}}$. Estos ciclos pueden ser de corto o largo plazo, por lo que no se realiza una descomposición de la serie en la forma usual de tendencia, ciclo, estacionalidad y componente irregular, sino que en su lugar se descompone la serie en la totalidad de frecuencias existentes.

$\mathrm{Si}$ se tiene un proceso estacionario con media $\boldsymbol{E}\left(\boldsymbol{Y}_{t}\right)=\boldsymbol{\mu}$ y autocovarianzas $\boldsymbol{E}\left(\boldsymbol{Y}_{t}-\boldsymbol{\mu}\right)\left(\boldsymbol{Y}_{t-j}-\boldsymbol{\mu}\right)=\boldsymbol{\gamma}_{j}$, $\mathrm{y}$ se asume que estas autocovarianzas son absolutamente sumables, la función de autocovarianzas generada se puede representar de la siguiente manera:

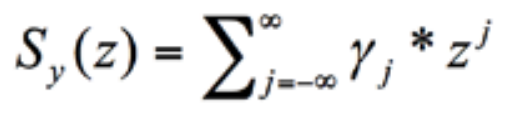


donde $\boldsymbol{z}$ es un escalar complejo. Si la expresión anterior se divide entre $2 \pi$ y se evalúa para $z=e^{-i w}$ donde $\boldsymbol{i}^{2}=-\boldsymbol{1}$ y $\boldsymbol{w}$ es un escalar real; es decir, se realiza una transformación de Fourier, el resultado es llamado el espectro poblacional de $\boldsymbol{Y}_{t}$ :

$S_{y}(w)=\frac{1}{2 \pi} \sum_{j=-\infty}^{\infty} \gamma_{j} * e^{-i w j}$

si adicionalmente, se utilizan algunos resultados trigonométricos, el espectro poblacional se puede reescribir como:

$$
S_{y}(w)=\frac{1}{2 \pi}\left[\gamma_{0}+\sum_{j=1}^{\infty} \gamma_{j} \cos (w j)\right]
$$

Esta es la representación espectral de la serie en el dominio de frecuencia e indica que la serie es igual a la suma de su promedio más la suma de un conjunto autocovarianzas de ciclos con frecuencias $\boldsymbol{w j}$.

En $S_{y}(\boldsymbol{w})$ se resume la variabilidad del proceso que generó la serie $\boldsymbol{Y}_{t}$, de la misma forma que en el dominio del tiempo este rol es jugado por la función de autocovarianzas. Por su forma de construcción, el espectro verifica las siguientes propiedades:

Si $\gamma_{j}$ constituyen las autocovarianzas de un proceso débilmente estacionario, entonces $\boldsymbol{S}_{\boldsymbol{y}}(\boldsymbol{w})$ es una función de valores reales, continua y no negativa.

El espectro es simétrico con respecto al origen de coordenadas, pues como $\boldsymbol{c o s}(\boldsymbol{w} \boldsymbol{j})=\boldsymbol{c o s}-\boldsymbol{w j}$ ) se tiene que $S_{y}(w)=S_{y}(-w)$.

El espectro es una función periódica respecto a $\boldsymbol{w}$ con período $\mathbf{2 \pi}$. Esto implica que el conocimiento de $S_{y}(\boldsymbol{w})$ para los $w \in[0, \pi]$ es suficiente para conocer el espectro en cualquier valor de $\boldsymbol{w}$.

Para entender la importancia y significación del espectro es interesante considerar la relación entre $S_{y}(\boldsymbol{w})$ (dominio de la frecuencia) y $\gamma_{j}$ (dominio del tiempo). Dicha relación está representada en la ecuación 4 que conforma la tesis principal del teorema Weiner-Kinchine para procesos estacionarios.

$$
\int_{-\pi}^{\pi} S_{y}(w) e^{-i w j} d w=\gamma_{j}
$$

La ecuación 4 muestra que la variabilidad de la serie está resumida en el espectro, en particular dice que a partir de $S_{y}(\boldsymbol{w})$ es posible obtener las autocovarianzas de la serie. Por otro lado la ecuación 3 define el espectro poblacional de la serie como una función de $\gamma_{j}$. En conjunto, ambas ecuaciones determinan la existencia de una relación biyectiva entre el espectro poblacional y la estructura de autocovarianzas, y por lo tanto determinan la equivalencia entre el dominio del tiempo y el dominio de la frecuencia para el estudio de series cronológicas.

Si se considera el caso particular cuando $\boldsymbol{j}=\boldsymbol{O}$, se puede ver que:

$$
\int_{-\pi}^{\pi} S_{y}(w) d w=\gamma_{0}
$$

Es decir que la variabilidad total de la serie es igual al área bajo el espectro poblacional. Además, como el espectro es siempre no negativo, la integral $\int_{w_{1}}^{w_{2}} S_{y}(w) d w$ puede ser interpretada como laporción de varianza explicada por periodicidades de frecuencias entre $\boldsymbol{w}_{1}$ y $\boldsymbol{w}_{2}$. Así, utilizando el espectro poblacional es posible descomponer la serie en componentes vinculados a periodicidades con distinto rango de frecuencias. Esto vincula al análisis espectral de la serie con la teoría de extracción de señales.

La estimación empírica del espectro poblacional a partir de una muestra finita es conocida como análisis espectral. Para ello, debe considerarse que para una muestra de tamaño $\mathrm{T}$, el ciclo más amplio que 
puede ser detectado en los datos es una frecuencia que completa un ciclo en toda la extensión de la serie. Además, el período más corto que se puede considerar o la frecuencia más alta es de 2 veces la unidad de medida del tiempo. La totalidad de frecuencias que se pueden observar son tantas como la mitad del número de observaciones.

\section{RESULTADOS Y DISCUSIÓN}

Se realizó un estudio por separado de las series de importaciones y exportaciones. Como puede apreciarse en la Figura 1, ambas series presentan una tendencia creciente; en consecuencia, en ambos casos se procedió inicialmente a transformar la serie en estacionaria eliminando la tendencia. La razón de eliminar la tendencia se basa en que dicha transformación filtra las frecuencias más bajas y permite tratar con más claridad las frecuencias altas.

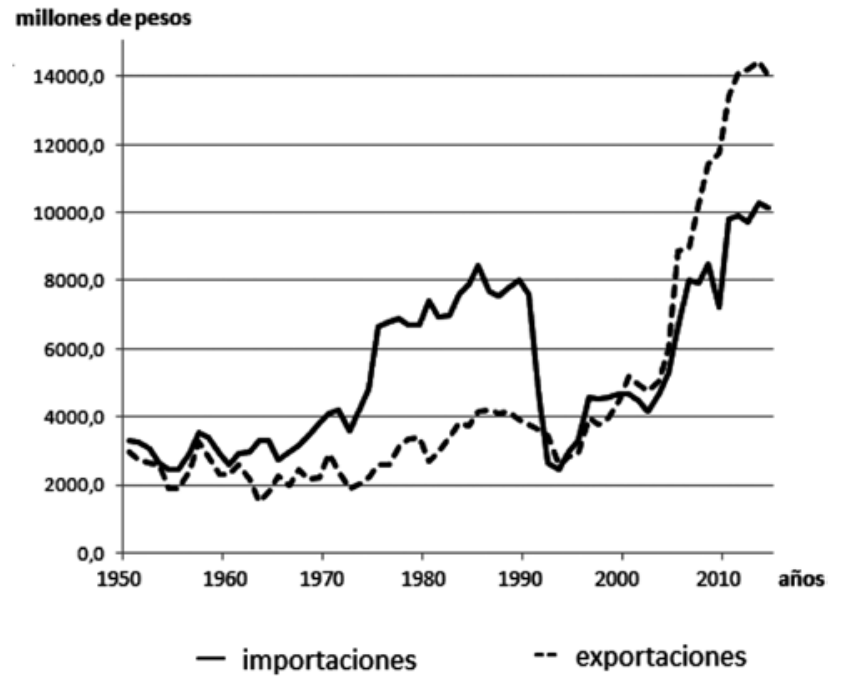

Fig. 1 Series de las importaciones y las exportaciones.

\section{Serie de importaciones}

Una vez obtenida la serie estacionaria se procedió a calcular su espectro, que puede verse en la Figura 2. Como la unidad de tiempo en las series originales es de un año, en el espectro la frecuencia está expresada en ciclos por años (cpa).

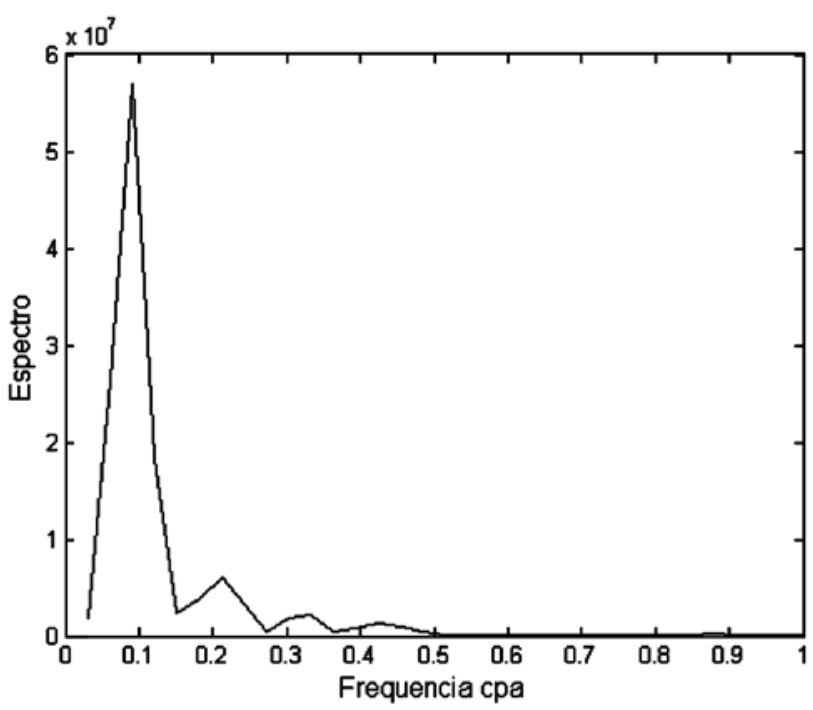

Fig. 2 Espectro de la serie de las importaciones.

Los picos deben entenderse como indicadores de componentes cíclicos con períodos constantes. Cada uno de los picos representa un tipo de ciclicidad diferente; cada una con una frecuencia determinada. En el gráfico se destacan tres ciclos con frecuencias bien definidas: una primera de $0.09 \mathrm{cpa}$, que corresponde a un periodo de 21.3 años; la segunda de $0.21 \mathrm{cpa}$, equivalente a 9.14 años y por último la de $0.34 \mathrm{cpa}$, que representa 5.8 ańos.

Una última frecuencia también se distingue $0.43 \mathrm{cpa}$ (periodo de 4.5 ańos) aunque esta con muy poco valor en el espectro.

\section{Serie de exportaciones}

En este caso una vez calculada el espectro, Figura 3, pueden apreciarse tres picos significativos. 


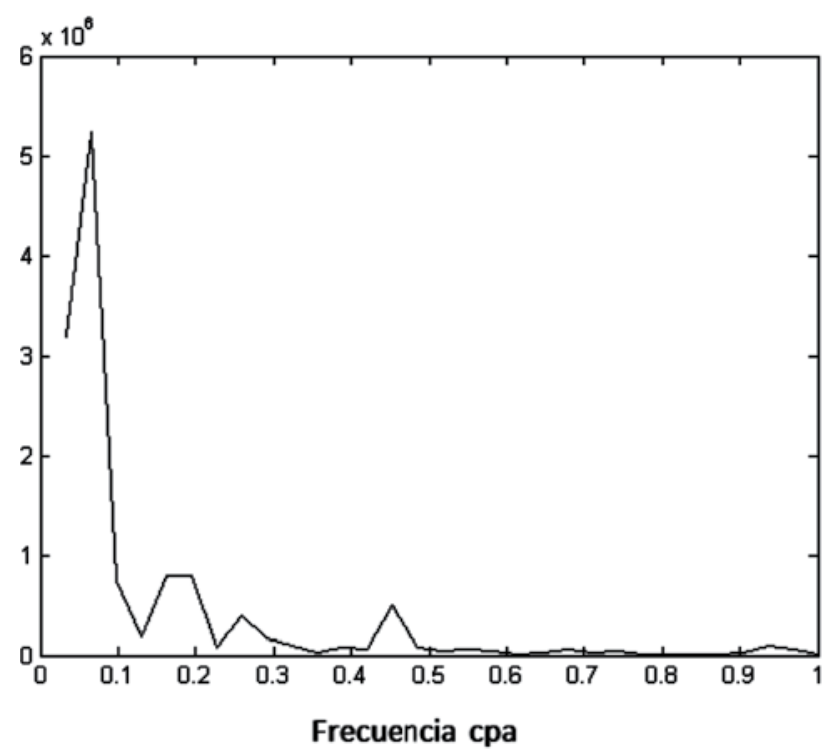

Fig. 3 Espectro de la serie de las exportaciones.

En primer término un pico importante para la frecuencia $0.06 \mathrm{cpa}$, periodo de 30 años; un segundo pico importante en la frecuencia de $0.43 \mathrm{cpa}$, periodo de 4.5 años y un tercer pico en la frecuencia de $0.25 \mathrm{cpa}$, periodo de 8 años.

Resumiendo, se puede apreciar que para ambas series se obtuvieron espectros con la forma característica de las series económicas, en los cuales los picos con las frecuencias significativas se encuentran en las frecuencias más bajas (Rodríguez, 2011). Es muy alentador observar que los espectros de ambas series presentan una determinada relación entre las frecuencias significativas, lo cual estaría sugiriendo que el análisis espectral está captando la esperada relación existente entre importaciones y exportaciones.

\section{Conclusiones}

1. Tanto para las importaciones como para las exportaciones fueron detectados tres ciclos significativos.

2. Los ciclos detectados en la serie de importaciones corresponden a periodos de 21, 9 y 6 años.

3. Los ciclos detectados en la serie de exportaciones corresponden a periodos de 30,8 y 5 años.

Como consideración final se puede expresar que aplicar el análisis espectral a las series de importaciones y exportaciones permitió conocer cuáles son sus ciclos determinísticos. Posibles pasos posteriores serian hacer pronósticos a corto plazo; así como estudiar la relación entre ambas series a través de los ciclos detectados.

\section{REFERENCIAS}

Cohen, D. (1999). An Analysis of Government Spendig in the Frequency Domain. Finance and Economics Discussion Series. Federal Reserve System.

González, D. (2001), Análisis espectral: consideraciones teóricas y aplicabilidad. Economía y Sociedad, 16, 45-60.

Granger, C. (1964). Spectral Analysis of Economic Time Series. Princeton NJ.: Princeton University Press.

Hamilton, J. (1994). Time Series Analysis. Princeton NJ.: Princeton University Press.

Jaramillo, V.D. (2010). Aplicación del análisis espectral de series temporales al modelo tricíclico de Schumpeter. Tendencias, $X I(2), 63-83$.

Priestley, M. (1981). Spectral Analysis and Time Series. Vol. I y II, New York: Academic Press, Inc.

Rodríguez, A. (2011). Análisis espectral de indicadores de precios en Costa Rica. Serie Documentos de Investigación No. 07-2011. Departamento de Investigación Económica. Banco Central de Costa Rica. 
